\title{
A generic method for the identification of options for cleaner products
}

\author{
Reinout Heijungs \\ Centre of Encironmental Science, Leiden University, P.O. Box 9518, 2300 RA Leiden, Netherlands
}

(Accepted 19 March 1993)

\begin{abstract}
Life cycle assessment, a method for the asscssment of the cnvironmental impacts of products, is briefly cxplaincd. A mathematical method to perform the calculations and to identify dominant aspects in the environmental load of a product is developed. The results are used to derive expressions for a marginal analysis which can be used for improvement analysis. In this way, a designer or process engineer can determine which processes or materials to consider first when (re)designing a product. The method developed can also be used to estimate the reliability of the determination of the environmental load of the products analyzed in terms of the reliability of the data of the processes involved.
\end{abstract}

Key words: Cleaner products; Improvement analysis; Life cycle assessment; Matrix sensitivity

\section{Introduction}

In the last two decades, environmental concern has become an important issue. Notions such as sustainability and industrial ecology have been proposed, first more or less as a metaphor, later in a more rigid definition. Many contributions to these subjects consider the interaction between the world's or a national economy and the environment. Sustainability inevitably deals with the effects of society as a whole (see, e.g., Common and Perrings, 1992). Ultimately, this is what the discipline of ecological economics is all about. The complexity of the relations between different parts of the world's economy and the environment is a problem, however. The analysis of environmental problems can sometimes be clarified by focusing on a restricted part of this complex of interwoven entities. A material balance approach, e.g., focusses on the routes of a substance or a group of substances within the economy and the environment (see, e.g., Ayres and Rod, 1986). Industrial ecology is concerned with the relation between different parts of the economic system (see, e.g., Patel, 1992).

In this paper we will choose another restriction on the analysis. We will concentrate on the environmental effects of products. The question of which of two product alternatives is to be preferred with respect to the environment is one which often instigates discussions, even when a quantitative analysis is made. For a recent example, see Hocking (1991) and reactions in the June 1991 issue of Science. An analysis which takes 
into account the entire life cycle of a product ("from cradle to grave") is called life cycle assessment (LCA). LCA is an analyzing tool for the assessment of the environmental impact of the functioning of a product. That the functioning of a product does not only include the usage of the product, but also the production, transportation, maintenance and waste handling, is reflected in the term life cycle. Some confusion may arise from the fact that the term, life cycle, is also in use by related fields of study where it has a somewhat different meaning, e.g., one frequently encounters a concept like the life cycle of a material in the context of integrated chain management. To avoid such complications, it is necessary to define the basic concepts of LCA. The subject of LCA is a given amount of service delivered by a product. The annual consumption volume of this service, its societal desirability or acceptability, and the relation between the product's effects and assumed thresholds of sustainability are not studied in an LCA. LCA is merely a tool within the framework of sustainability. Among possible applications are the comparison of product alternatives and the (re)design of products in an environmentally optimal way. Internalization of environmental costs is conceivable, but requires the monetary valuing of environmental effects.

Life cycle assessment is a rapidly developing subject of study (Fava et al., 1991; Heijungs et al., 1992; Anonymous, 1992; Vigon et al., 1992). The methodology still poses interesting theoretical and practical problems, most of which will not be discussed within this paper. Despite the obscurities (see Guinée et al., 1993), LCAs are performed within many countries and by many companies.

This paper provides explicit algebraic equations to solve the computational problems associated with LCA (section 3). The differences between the proposed method and other methods are discussed (section 5). The analytic expressions derived can be used to provide information on options for the environmental improvement of a product (section 6). Thus the designer is informed about how to diminish the environmental load of the life cycle of a product with the least effort. It remains a task for the designer to use his or her creativity in the investigation of alternative processes or materials, or to modify the characteristics of processes, in order to decrease the environmental load. Additionally, formulae will be derived which can be used to estimate the reliability of the calculation of the environmental load of the life cycle of a product (section 8). This is an important aspect since inaccurate data may corrupt a life cycle assessment in a so far unknown way. Examples in sections 4, 7 and 9 illustrate the usage of the theory.

\section{Life cycle assessment}

One of the recent developments within the field of LCA is a fairly general agreement on the structure (SETAC-Europe workshop, 1992). An LCA is only concerned with the environmental aspects of a product; other aspects, such as costs or convenience, are excluded. Eventually an exhaustive assessment is madc, including all relcvant aspects. In an LCA five components can be distinguished: goal definition, inventory, classification, evaluation ${ }^{1}$ and improvement analysis.

In the goal definition, the subject of study is determined. This includes a description of the amount of function investigated, the so-called functional unit. An example of a functional unit is "packaging of a sandwich". Using this functional unit, packaging systems of different materials (polyethene, aluminium, paper, etc.) can be compared.

The next component of an LCA is the inventory. Within the inventory, the life cycle of each of the products considered is defined by assembling the processes which constitute the different phases of the life cycle. These phases consist of industrial processes such as the production of materials or components, consumer processes such as the use and maintenance of products, and

\footnotetext{
1 Sometimes, classification and evaluation are treated as a unitary component under the name impact analysis. There is also some controversy whether the name valuation or evaluation is to be preferred.
} 
post-consumer processes such as waste handling and recycling. The collection of combined processes with their mutual relationship is called a process tree. The inventory also comprises the collection of the process data. Process data consist of economic data (use and production of materials, products and services) and of environmental data (extractions of resources and emissions of substances). The calculation of the quantitative contribution of every process to the process tree is a computational task. The result of the inventory is a list of the loading onto the environment in terms of extractions and emissions caused by a functional unit of the product analyzed.

In the classification, scientific knowledge of environmental processes is used to estimate the contribution of all extractions and emissions to a limited number of generally recognized environmental problems. For example, emissions of $\mathrm{SO}_{2}$, $\mathrm{NO}_{x}$ and $\mathrm{NH}_{x}$ are aggregated according to their potential contribution to acidification. This is achieved by multiplying those emissions by a conversion factor which represents the potency to create $\mathrm{H}^{+}$-ions per $\mathrm{kg}$ of the substance.

In practice one product alternative will seldom be preferred over the other in all environmental aspects. Thence the need for an evaluation, in which the relative importance of each of the environmental problems is assessed. The evaluation facilitates a decision on the choice between product alternatives, or on the subject of product improvement.

An LCA can be regarded as a decision support system. One may think of decisions on the purchase of products, on policy measures like "ecolabelling", on "green marketing" or on the (re)design of products as being based on the information of an LCA. These decisions are outside the scope of an analytical tool such as LCA. However, the methodological aspects, notably concerning the identification of options for improvement, are part of an LCA. The framework of LCA, therefore, includes an improvement analysis, which may be skipped depending on the application.

The components - goal definition, inventory, classification and evaluation - all yield informa- tion on the interaction between the product and the environment. The information from different components is mutually dependent: the inventory gives, e.g., the quantified emissions of all greenhouse gases, whereas the result of the classification contains a number that denotes the contribution of those emissions to global warming. Therefore, one has to choose a level of assessment. In many studies this is the inventory. Somewhat more disputed is a description at the level of the classification, let alone the evaluation. Much current research is devoted to the problems of implementing a classification and the possible solutions to these problems. In most studies the evaluation has received little or no attention.

The improvement analysis is the only component which does not yield a description of the interaction between life cycle and environment; it gives information on the opportunities to decrease the environmental burdens associated with a functional unit of product. One is free to choose whether an improvement analysis is undertaken after the inventory, after the classification or after the evaluation. In the rest of this paper, some aspects of the inventory and the improvement analysis succeeding the inventory are discussed. We deliberately give a conservative approach, and try to omit discussions on the usefulness and feasibility of classification and valuation.

\section{Calculations within the inventory}

Given a process tree, data on the processes involved are collected. These data are usually specified per standard unit, such as per $1000 \mathrm{~kg}$ steel. Therefore, the quantitative occurrence of the processes in the process tree under study must be calculated.

Processes may be represented in vector notation and will be handled in a way which resembles the classical input-output analysis (for an example of its usage in economic considerations, see Leontief (1986); for an extension to environmental aspects, see Duchin (1992)). A process has two characteristic parts: the economic part and the environmental part. The economic part contains information on the use and production of 
economic entities. Economic entities are entities which are being dealt with in (and, therefore, are inputs from and outputs to) other processes. Examples of economic entities are materials, products, services, energy, and waste to be processed. They are thus opposed to environmental entities, which are extracted from, resp. emitted to the environment without previous resp. further processes. A process can be described with a number $r$ of economic entities $a_{j}$ and a number $s$ of environmental entities $b_{k}$ as

$$
\left(\begin{array}{l}
\boldsymbol{a} \\
\boldsymbol{b}
\end{array}\right)=\left(\begin{array}{c}
a_{1} \\
\ldots \\
a_{j} \\
\ldots \\
a_{r} \\
b_{1} \\
\ldots \\
b_{k} \\
\ldots \\
b_{s}
\end{array}\right)
$$

where $a_{1}$ could denote "use of $3 \mathrm{~kg}$ PVC", $a_{2}$ "production of $12 \mathrm{MJ}$ electricity", $b_{1}$ "extraction of $2.6 \mathrm{~kg}$ iron ore" and $b_{2}$ "emission of $15 \mathrm{~g} \mathrm{SO}_{2}$ ". A sign convention will be adopted: inputs ("use of", "extraction of") will be expressed by negative coefficients and outputs ("production of", "emission of') by positive coefficients.

Let every entity have an additional subscript which indicates the process it characterizes, so let $a_{j i}$ denote the $j$ th economic entity of process $i$ and $b_{k i}$ the $k$ th environmental entity of process $i$. Suppose that the process tree consists of $q$ different processes. All process characteristics of the process tree can now be represented by a matrix of dimension $(r+s) \times q$ :

$$
\left(\begin{array}{c}
\boldsymbol{A} \\
\boldsymbol{B}
\end{array}\right)=\left(\begin{array}{ccccc}
a_{11} & \ldots & a_{1 i} & \ldots & a_{1 q} \\
\ldots & \ldots & \ldots & \ldots & \ldots \\
a_{j 1} & \ldots & a_{j i} & \ldots & a_{j q} \\
\ldots & \ldots & \ldots & \ldots & \ldots \\
a_{r 1} & \ldots & a_{r i} & \ldots & a_{r q} \\
b_{11} & \ldots & b_{1 i} & \ldots & b_{1 q} \\
\ldots & \ldots & \ldots & \ldots & \ldots \\
b_{k 1} & \ldots & b_{k i} & \ldots & b_{k q} \\
\ldots & \ldots & \ldots & \ldots & \ldots \\
b_{s 1} & \ldots & b_{s i} & \ldots & b_{s q}
\end{array}\right)
$$

A special kind of process is the process of which the functional unit is an output. That is, there exists a process that is the aggregation of both economic and environmental entities of the process tree: a process which has no economic inputs and outputs but the delivery of the functional unit, and which has a hitherto unknown environmental part. It will be called the kernel process and will be denoted by a column vector of dimension $(r+s)$ which also consists of an economic part $\boldsymbol{\alpha}$ and an environmental part $\boldsymbol{\beta}$ :

$$
\left(\begin{array}{c}
\boldsymbol{\alpha} \\
\boldsymbol{\beta}
\end{array}\right)=\left(\begin{array}{c}
\alpha_{1} \\
\ldots \\
\alpha_{j} \\
\ldots \\
\alpha_{r} \\
\beta_{1} \\
\ldots \\
\beta_{k} \\
\ldots \\
\beta_{s}
\end{array}\right)
$$

As stated, in practice all $\alpha_{j}$ will be 0 except for one. In the example mentioned, the only non-zero economic entity of the kernel process is "packaging of a sandwich". The environmental entities of the kernel process are the environmental inputs and outputs associated with a functional unit $\boldsymbol{\alpha}$ and, therefore, unknown; they pose the central quest in the inventory of an LCA.

The quantitative occurrence of a process $i$ contained in the process tree will be called $p_{i}$. It can be calculated using a physical (mass or energy) balance: whenever a process uses a certain amount of a certain economic entity, it is delivered by the other processes in the process tree in cxactly the amount required. The converse is also true: whenever a process produces a certain amount of a certain economic entity, it is used by the other processes in exactly the amount given. ${ }^{2}$ The algebraic statement of the law of conserva-

\footnotetext{
${ }^{2}$ This mass or energy balance per entity is not the same as the balance within a process (law of Lavoisier); the latter (which can be used on an overall mass basis or at a molecular level) is not necessary for the validity of the equations derived in this paper, but will often be used in the assessment of the quality of the process data.
} 
tion of mass and energy for entity $j$ reads

$$
\sum_{i=1}^{q} a_{j i} p_{i}=\alpha_{j} \text {. }
$$

This should hold simultaneously for all economic entities $(j=1, \ldots, r)$ :

$\forall j=1, \ldots, r: \quad \sum_{i=1}^{q} a_{j i} p_{i}=\alpha_{j}$.

Eq. 1 will be called the balance equation.

We will assume that all process are single processes, i.e., that all processes serve one purpose. In reality therc arc many multiple processes, e.g., the co-production of chlorine and caustic soda. The economic and environmental parts of such processes will have to be distributed over the multiple outputs: chlorine and caustic soda. The details of this distribution, which is generally called allocation, is a subject of other studies (see, e.g., Huppes, 1992). One of the consequences of allocation is that the number of processes $q$ is equal to the number of economic entities $r$ :

$q=r$

so that the matrix $A$ is square. In this paper, however, the notational distinction between $q$ and $r$ is maintained for the sake of clarity.

The balance equation can be solved for $p_{i}$ using Cramer's rule, which states that for a square non-singular matrix

$p_{i}=\frac{\operatorname{det}\left(A^{i}\right)}{\operatorname{det}(A)}, \quad i=1, \ldots, q$

where $A^{i}$ is equal to the matrix $A$ with the $i$ th column replaced by the vector $\alpha$ and $\operatorname{det}(A)$ denotes the determinant of the matrix $A$. Since Cramer's rule requires the matrix to be square, one "proves" mathematically that single processes should be used when the process tree is drawn up. The matrix will be singular if and only if there is a linear dependency of two or more columns. This may happen if, e.g., a non-elementary process is defined together with its constituent processes. The data redundancy can be avoided by skipping such "double" process definitions.
When the environmental part of every process is multiplied by the quantitative occurrence of that process, the environmental part $\boldsymbol{\beta}$ of the kernel process can be obtained by aggregating over all processes involved:

$\beta_{k}=\sum_{i=1}^{q} b_{k i} p_{i}, \quad k=1, \ldots, s$.

We now define the process matrix $\boldsymbol{P}$ of dimension $(r+s) \times(q+1)$ as

$\boldsymbol{P}=\left(\begin{array}{rr}\left(a_{j l} p_{i}\right)_{i=1, \ldots, q} & \left(\alpha_{j}\right)_{j=1, \ldots, r} \\ j=1, \ldots, r & \\ \left(b_{k i} p_{\imath}\right)_{i=1, \ldots, q} & \left(\beta_{k}\right)_{k=1, \ldots, s} \\ k=1, \ldots, s & \end{array}\right)$.

In this compact notation, substitution of Eqs. 2 and 3 is implied. This matrix gives a complete overview of the amount to which every economic and environmental entity is involved. This makes it possible to perform a dominance analysis; one can study which processes contribute dominantly to a particular emission.

\section{Example 1}

As an example, consider a hypothetical process tree based on the four processes $(q=r=4)$ with four environmental entities $(s=4)$ in Table 1.

The economic part $A$ of the process tree can be represented by the matrix

$A=\left(\begin{array}{crrr}1 & -50 & -1 & 0 \\ -0.01 & 1 & -1 & 0 \\ 0 & 0 & 1 & -1 \\ 0 & 0 & 0 & 1\end{array}\right)$

and the environmental part $B$ by

$\boldsymbol{B}=\left(\begin{array}{crrr}0 & -5 & 0 & 0 \\ -0.5 & 0 & 0 & 0 \\ 3 & 0 & 0 & 0 \\ 2 & 10 & 0 & 1\end{array}\right)$

The economic part $\alpha$ of the kernel process is given by the vector

$\boldsymbol{\alpha}=\left(\begin{array}{l}0 \\ 0 \\ 0 \\ 0.1\end{array}\right)$ 
Table 1

Example of some processes which constitute a hypothetical process tree for the packaging of a sandwich. Notice that there is a certain amount of aluminium required for the production of electricity. The kernel process is not equal to the consumer process; the functional unit is contained in both

\begin{tabular}{lccccc}
\hline entity & \multicolumn{2}{l}{ process } & & & \multirow{2}{*}{ kernel process } \\
\cline { 2 - 5 } & $\begin{array}{l}\text { production of } \\
\text { electricity }\end{array}$ & $\begin{array}{l}\text { production of } \\
\text { aluminium }\end{array}$ & $\begin{array}{l}\text { production of } \\
\text { aluminium foil }\end{array}$ & $\begin{array}{l}\text { usage of } \\
\text { aluminium foil }\end{array}$ \\
\hline MJ electricity & 1 & -50 & -1 & 0 & 0 \\
kg aluminium & -0.01 & 1 & -1 & 0 & 0 \\
kg aluminium foil & 0 & 0 & 1 & -1 & 0 \\
100 sandwich packages & 0 & 0 & 0 & 0 & 0.1 \\
kg bauxite & 0 & -5 & 0 & 0 & $?$ \\
kg crude oil & -0.5 & 0 & 0 & 0 & $?$ \\
kg CO & 3 & 10 & 0 & 1 & $?$ \\
kg solid waste & 2 & & 0 & $?$
\end{tabular}

The determinant $\operatorname{det}(A)=0.5 ; \operatorname{det}\left(A^{i}\right)$ are (for $i=1, \ldots, 4) 5.1,0.101,0.05$ and 0.05 and the coefficients $p_{i}$ are $10.2,0.202,0.1$ and 0.1 . One can easily check that the coefficients $p_{i}$, calculated with Eq. 2 satisfy the balance equation (Eq. 1) with the process data from Table 1 . The environmental part $\boldsymbol{\beta}$ of the kernel process is readily calculated as

$\boldsymbol{\beta}=\left(\begin{array}{c}-1.01 \\ -5.1 \\ 30.6 \\ 22.52\end{array}\right)$

This is the required result of the inventory: these numbers represent the environmental load of a functional unit, the question marks in Table 1. These are: extraction of $1.01 \mathrm{~kg}$ bauxite and 5.1 $\mathrm{kg}$ crude oil, emission of $30.6 \mathrm{~kg}$ carbondioxide and the disposal of $22.52 \mathrm{~kg}$ of solid waste. This can be further assessed in the classification.

The process matrix $\boldsymbol{P}$ is given by

$$
P=\left(\begin{array}{ccccc}
10.2 & -10.1 & -0.1 & 0 & 0 \\
-0.102 & 0.202 & -0.1 & 0 & 0 \\
0 & 0 & 0.1 & -0.1 & 0 \\
0 & 0 & 0 & 0.1 & 0.1 \\
0 & -1.01 & 0 & 0 & -1.01 \\
-5.1 & 0 & 0 & 0 & -5.1 \\
30.6 & 0 & 0 & 0 & 30.6 \\
20.4 & 2.02 & 0 & 0.1 & 22.52
\end{array}\right) .
$$

A dominance analysis shows, for example, that about $90 \%$ of the amount of waste is produced during the production of electricity. Hence the production of electricity is a dominant aspect for solid waste production.

\section{Differences from other methods}

This section will explain the differences between the matrix method introduced with Eqs. 1 and 2 and other methods to solve the process tree from an LCA.

The most straightforward approach is to consider the functional unit as the starting point and to collect data in the right amount per entity (substance, energy, service), such that a balance equation similar to Eq. 1 is satisfied. This is a sequential method: one searches and adds multiples of processes until there is no economic entity unsatisfied. Since most methods for LCA do not mention a method to solve the process tree, it is assumed here that the sequential method is the method most often used.

The existence of self-referring groups of processes, such as is the case in the production of electricity and of coke, poses problems with this approach. The experimental computer program SIMAPRO (Goedkoop, 1991) is not resistant against such networks: a program crash (stack overflow) will occur. In a book on life cycle inventories (Fava et al., 1991), it is suggested that "the only satisfactory way of dealing with such net- 
works is iteration". Iteration is sustained until some criteria of convergence are met. This implies a number of runs which is unknown beforehand, and the introduction of additional inexactness. The report of the IDEA-project (Lübkcrt ct al., 1991) gives more detailed criteria for convergence in terms of a tolerance parameter $\zeta$.

The method which solves a system of equations simultaneously, as is the case in the balance equation 1 , will be called the matrix method. The matrix method has the advantage that networks with mutually referring processes can be handled exactly and without special treatment such as iteration.

At one of the Fraunhofer-Instituts (1991), a method has been developed which resembles the method proposed in this paper. Three differences hetween the two approaches will be mentioned. The first is that the method proposed by the Fraunhofer-Institut does not make a distinction hetween the economic and the environmental part of the processes. Instead they define so-called formal processes which are a mathematical trick to guarantee the matrix of the process tree will be a square matrix. A consequence of this is that the matrix $\boldsymbol{A}$ is not of dimension $r \times r$, but of dimension $(r+s) \times(r+s)$, which implies an increased demand on computer capacity.

The second difference is that in solving the equations, the inverse of the matrix is calculated instead of the determinant. We presume that the determinant of the matrix is more easily calculated than the inverse of the matrix. Moreover, the expression in terms of a determinant offers the possibility to give analytical expressions for a sensitivity analysis; see sections 6 and 8 .

The third difference is the absence of an explicit kernel process in the calculation; a process that delivers the functional unit is included in the matrix $\boldsymbol{A}$ and the process tree does not deliver an external function as is the case in Eq. 1. This means that one of the columns of the matrix has to be fixed in an artificial way. The advantage of the method proposed in this paper is that a life cycle has a very explicit result: the kernel process, not only the economic part (the functional unit) but also the environmental part. After completing the environmental part $\boldsymbol{\beta}$ the kernel process can be regarded as a ready-to-use function delivering process, which may be the input of another process in another process tree.

\section{Marginal analysis}

The matrix method gives an explicit expression for the environmental part of the functional unit. This expression can be used to deduce formulae for a sensitivity analysis. The next sections will discuss two applications: a reliability analysis (section 8) and a marginal analysis (this section).

In a marginal analysis, one studies the effects of small changes on the results. This can be used to enable improvement analysis: processes to modify can be preselected using knowledge of the sensitivity of the result to small perturbations in the economic or environmental process data. A designer or process engineer is informed on the marginal yields of interferences in the characteristics of individual processes.

Assume that an cconomical process characteristic $a_{j i}$ is modified. This implies a change in the occurrence of the processes $p_{l}(l=1, \ldots, q)$, and thus of the environmental load $\beta_{k}(k=1, \ldots, s)$. The change of $\beta_{k}$ due to a change of only $a_{j i}$ is denoted by $\Delta \beta_{k}\left(\Delta a_{j i}\right)$ and is in a first-order approximation given by

$\Delta \beta_{k}\left(\Delta a_{j i}\right)=\frac{\partial \beta_{k}}{\partial a_{j i}} \Delta a_{j i}$

For reasons that will become obvious, we will derive relative relations, so not in terms of $\Delta \beta_{k}\left(\Delta a_{j i}\right)$, but in terms of $\Delta \beta_{k} / \beta_{k}\left(\Delta a_{j i} / a_{j i}\right)$. In the appendix, it is found that

$$
\begin{aligned}
\frac{\Delta \beta_{k} / \beta_{k}}{\Delta a_{j i} / a_{j i}}= & -a_{j i} \frac{(-1)^{j+i} \operatorname{det}\left(A_{j i}\right)}{\operatorname{det}(A)} \\
& +\frac{a_{j i}(-1)^{j+i}}{\beta_{k} \operatorname{det}(A)} \sum_{\substack{l=1 \\
l \neq i}}^{q}\left(b_{k l} \operatorname{det}\left(A_{j i}^{l}\right)\right),
\end{aligned}
$$

where $\boldsymbol{A}_{j i}$ denotes the matrix $\boldsymbol{A}$ with the $j$ th row and the $i$ th column deleted (the minor) and $A_{j i}^{l}$ the minor of the matrix $\boldsymbol{A}^{l}$. 
For the change of $\beta_{k}$ due to a change of one of the $\alpha_{j}$ we will write $\Delta \beta_{k} / \beta_{k}\left(\Delta \alpha_{j} / \alpha_{j}\right)$. One derives for the relative change that

$$
\frac{\Delta \beta_{k} / \beta_{k}}{\Delta \alpha_{j} / \alpha_{j}}=\frac{\alpha_{j}}{\beta_{k}} \sum_{i=1}^{q}\left(\frac{(-1)^{j+i} \operatorname{det}\left(A_{j i}\right)}{\operatorname{det}(\boldsymbol{A})} b_{k i}\right) \text {. }
$$

It can be proven that if only one element of $\boldsymbol{\alpha}$, say $\alpha_{j}$, is non-zero, the right hand side of Eq. 6 is reduced to zero for all $j \neq m$, and is unity for $j=m$, so that $\beta_{k}$ is proportional to $\alpha_{j}$ as should be expected. A more complicated situation arises when multiple functional units are used.

The expressions for changes in the environmental entities are easier: the quantitative occurrence $p_{l}$ of the processes is unaltered. For $\Delta \beta_{k} / \beta_{k}\left(\Delta b_{n i} / b_{n i}\right)$ one finds

$$
\frac{\Delta \beta_{k} / \beta_{k}}{\Delta b_{n i} / b_{n i}}= \begin{cases}\frac{b_{k i} p_{i}}{\beta_{k}} & (\text { if } n=k) \\ 0 & \text { (otherwise) }\end{cases}
$$

and (a little superfluous; only for reasons of symmetry) for $\Delta \beta_{k} / \beta_{k}\left(\Delta \beta_{n} / \beta_{n}\right)$

$$
\frac{\Delta \beta_{k} / \beta_{k}}{\Delta \beta_{n} / \beta_{n}}= \begin{cases}1 & (\text { if } n=k) \\ 0 & \text { (otherwise) }\end{cases}
$$

For a particular choice of $k$ one can construct a $(r+s) \times(q+1)$-dimensional matrix $D_{k}$, the marginal matrix, again a shorthand form in which Eqs. 5 to 7 are to be substituted:

$$
\boldsymbol{D}_{k}=\left(\begin{array}{ll}
\left(\frac{\Delta \beta_{k} / \beta_{k}}{\Delta a_{j i} / a_{j i}}\right)_{i=1 \ldots . . q} & \left(\frac{\Delta \beta_{k} / \beta_{k}}{\Delta \alpha_{j} / \alpha_{j}}\right)_{j=1, \ldots . r} \\
\left(\frac{\Delta \beta_{k} / \beta_{k}}{\Delta b_{n i} / b_{n i}}\right)_{\substack{i=1, \ldots . q \\
n=1 \ldots ., s}} & \left(\frac{\Delta \beta_{k} / \beta_{k}}{\Delta \beta_{n} / \beta_{n}}\right)_{n=1, \ldots, s}
\end{array}\right),
$$

which indicates the relative sensitivity of $\beta_{k}$ to a small change in one of the process characteristics $a_{j i}, b_{n i}$ or $\alpha_{j}$. That is to say, the symbolic notation

$$
\left(\frac{\Delta \beta_{k}}{\beta_{k}}\right)=\left(D_{k}\right)_{j i}\left(\frac{\Delta p_{j i}}{p_{j i}}\right) \text {, }
$$

where $p_{j i}$ indicates the element with entry $(j, i)$ of the process matrix $\boldsymbol{P}$, holds to first order. The marginal matrix can be interpreted as a matrix containing magnification factors for changes in process characteristics $p_{j i}$. For example, let the entry of the first row and the first column be 9. A change of the value of the first economic entity of the first process of $2 \%$ signifies a change of the chosen environmental entity of about $18 \%$. Obviously, a large number in $D_{k}$ denotes a process characteristic in which a small change will have significant effects. Conversely, a small number denotes a process characteristic which should not be focused on as a first priority. In this way, the designer is aided by his attention being drawn to the most sensitive process characteristics. Using this knowledge, processes can be readjusted or redesigned, or alternative processes or materials can bc uscd.

\section{Example 2}

Using the same process tree as in section 4 , the options for waste prevention $(k=4)$ are investigated. Application of section 6 gives for the marginal matrix $D_{4}$ :

$D_{4}=\left(\begin{array}{lllll}-1.902 & 1.883 & 0.019 & 0 & 0 \\ 0.996 & -1.973 & 0.977 & 0 & 0 \\ 0 & 0 & -0.996 & 0.996 & 0 \\ 0 & 0 & 0 & -1.000 & 1 \\ 0 & 0 & 0 & 0 & 0 \\ 0 & 0 & 0 & 0 & 0 \\ 0 & 0 & 0 & 0 & 0 \\ 0.906 & 0.090 & 0 & 0.004 & 1\end{array}\right)$.

The largest elements of this matrix are listed in Table 2. The following can be seen from the matrix or table:

- The easiest ${ }^{3}$ way is provided by modifying the coefficients $a_{11}$ (the production of electricity), $a_{22}$ (the production of aluminium) and $a_{12}$ (use of electricity by the aluminium production); an increase in the efficiency of one of those processes of $1 \%$ leads to a waste reduction of almost $2 \%$.

- Other possibilities are a decrease of the aluminium input of the electricity production $\left(a_{21}\right)$, a decrease of the amount of aluminium needed for foil production $\left(a_{23}\right)$ and a decrease of the amount of aluminium foil required for one sandwich $\left(a_{34}\right)$.

\footnotetext{
$\overline{3}$ Easiest has a very specific meaning here: its meaning will be clarified in the rest of the text.
} 
Table 2

The largest elements of the marginal matrix $D_{k}$ in the example of waste reduction $(k=4)$ for the packaging of a sandwich, using the process data of Table 1

\begin{tabular}{lllr}
\hline$p_{j 1}$ & process & entity & magnification \\
\hline$a_{z 2}$ & production of aluminium & kg aluminium & -1.973 \\
$a_{11}$ & production of electricity & MJ electricity & -1.902 \\
$a_{12}$ & production of aluminium & MJ electricity & 1.883 \\
$a_{44}$ & usage of aluminium foil & 100 sandwich packages & -1.000 \\
$a_{21}$ & production of electricity & kg aluminium & 0.996 \\
$a_{33}$ & production of aluminium foil & kg aluminium foil & -0.996 \\
$a_{34}$ & usage of aluminium foil & kg aluminium foil & 0.996 \\
$a_{23}$ & production of aluminium foil & kg aluminium & 0.977 \\
$b_{41}$ & production of electricity & kg solid waste & 0.906 \\
\hline
\end{tabular}

- Other somewhat less interesting and trivial options are an increase of the efficiency of the aluminium foil production $\left(a_{33}\right)$, and an increase of the number of sandwiches packed with the same amount of aluminium $\left(a_{44}\right)$.

- The last interesting option is the reduction of the amount of waste in the production of electricity $\left(b_{41}\right)$; an effort of $1 \%$ is paid back as about $0.9 \%$.

It is clear that the feasibility of these options must be judged by an expert, e.g., a process engineer, chemical engineer or designer. For examplc, the suggestion to increase the efficiency of the production of aluminium foil is not a very practical one; one cannot produce more than $1 \mathrm{~kg}$ of foil by using $1 \mathrm{~kg}$ aluminium. Aspects concerning cost-efficiency are also excluded in this part of the analysis. This method merely suggests what to consider as possibly relevant options. The resulting list of options should thereafter be judged on technical and financial feasibility. Direct combination of the marginal analysis and these aspects of feasibility is, however, possible and promising.

Notice that there is an important difference between a marginal analysis using the marginal matrix (Eq. 9), and a dominance analysis using the process matrix (Eq. 4). The dominance analysis in section 5 shows that the production of electricity is the major cause for the production of waste, and that the production of aluminium contributes to only $10 \%$ of the amount of waste. The marginal analysis suggests that a reduction in the electricity requirement of the production of aluminium has significant effects on the amount of waste. Study of the process matrix will eventually reveal the same, but the marginal analysis offers a very explicit statement.

\section{Reliability analysis}

When reliability estimates of process characteristics are known, the partial derivative deduced in the section on the dominance analysis (section 6) can be used to obtain an expression for an estimate of the reliability of $\beta_{k}$ in terms of the reliability of $\boldsymbol{a}, \boldsymbol{b}$ and $\boldsymbol{\alpha}$. The main mathematical difference is caused by the fact that all coefficients $a_{j i}, b_{k i}$ and $\alpha_{j}$ are now allowed to change simultancously.

Assume the process data are specified in the form $a_{j i} \pm \Delta a_{j i}, b_{k i} \pm \Delta b_{k i}$ and $\alpha_{j} \pm \Delta \alpha_{j}$. The environmental part of the functional unit is given by $\beta_{k} \pm \Delta \beta_{k} ; \Delta \beta_{k}$ follows to first order by using the definition of $\beta_{k}$ (Eq. 3):

$\Delta \beta_{k}=\sum_{l=1}^{q}\left(b_{k l} \Delta p_{l}+p_{l} \Delta b_{k l}\right)$ 
Using the results derived in section 6 , one finds for $\Delta p_{l}$

$$
\begin{aligned}
\Delta p_{l}= & \sum_{j=1}^{r}\left(\left(-p_{l} \sum_{i=1}^{q} \frac{(-1)^{j+1} \operatorname{det}\left(A_{j i}\right)}{\operatorname{det}(A)} \Delta a_{j i}\right)\right. \\
& +\left(\sum_{\substack{i=1 \\
i \neq l}}^{q} \frac{(-1)^{j+i} \operatorname{det}\left(A_{j i}^{l}\right)}{\operatorname{det}(A)} \Delta a_{j i}\right) \\
& \left.+\left(\frac{(-1)^{j+l} \operatorname{det}\left(A_{j l}\right)}{\operatorname{det}(A)} \Delta \alpha_{j}\right)\right),
\end{aligned}
$$

which can be substituted in Eq. 10. By defining $\Delta\left(a_{j i} p_{i}\right)=a_{j i}\left(\Delta p_{i}\right)+\left(\Delta a_{j i}\right) p_{i}$

and

$\Delta\left(b_{k i} p_{i}\right)=b_{k i}\left(\Delta p_{i}\right)+\left(\Delta b_{k i}\right) p_{i}$, one can define the reliability matrix $\Delta \boldsymbol{P}$ by

$\Delta \boldsymbol{P}=\left(\begin{array}{rl}\left(\Delta\left(a_{j i} p_{i}\right)\right)_{i}=1, \ldots, q & \left(\Delta \alpha_{j}\right)_{j=1, \ldots, r} \\ j=1, \ldots, r & \\ \left(\Delta\left(b_{k i} p_{i}\right)\right)_{i}=1, \ldots, q & \left(\Delta \beta_{k}\right)_{k=1, \ldots, s} \\ k=1, \ldots, s & \end{array}\right)$

so that the symbolic notation

$\boldsymbol{P} \pm \Delta \boldsymbol{P}$

makes sense. At present, data on confidence are mostly lacking so that many reliability estimates will be assumed to be zero. Hopefully, in the future such data will be known for many processes so that an estimate of the reliability will make more sense.

\section{Example 3}

Using the same processes and kernel process $(\boldsymbol{A}, \boldsymbol{B}$ and $\boldsymbol{\alpha})$ as in section 5 , assume the process characteristic $a_{12}$ (electricity . demand of aluminium production) to be known as $-50 \pm 5$. Using the reliability analysis of section 8 one finds

$$
\boldsymbol{\beta}=\left(\begin{array}{c}
-1.01 \pm 0.101 \\
-5.1 \pm 1.01 \\
30.6 \pm 6.06 \\
22.52 \pm 4.24
\end{array}\right)
$$

It is obvious that an inaccuracy of $10 \%$ can be enhanced for many aspects. This is, of course, reflected in the values of $\left(D_{k}\right)_{12}$, which is 1.00 , $1.98,1.98$ and 1.88 for $k=1, \ldots, 4$, respectively. Hence the need for a reliability analysis, at least for the "sensitive" parameters, such as $a_{12}$. Inaccuracies in other parameters, notably of the environmental part of processes, may lead to a diminished inaccuracy of the resulting $\boldsymbol{\beta}$, but still are worth assessing.

\section{Discussion}

The theory exposed above needs further elaboration, not only in hypothetical examples, but in real case studies. Computer implementation of Cramer's rule, the process matrix and the marginal matrix is straightforward. Implementation is also essential for the practical usage of the marginal matrix. The examples of this paper have becn calculated using a prototype of such a computer program. There are many methods to solve a system of linear equations. Except for the two mentioned thus far (Cramer's rule and matrix inversion), one can exploit Gauss-Jordan elimination, LU-decomposition, eigenvalues and so on. Which method to prefer depends on the properties of the matrix, e.g., whether it is ill-conditioned, is in block-diagonal form, or contains many zeros, as is the case in LCA. It is only for theoretical reasons that this paper uses determinants: partial derivatives needed for the derivation of the marginal matrix can be found using elementary calculus. Perhaps a computer program for LCA may better use an alternative method for computational reasons.

The extension of these ideas to the classification and the evaluation is mathematically trivial and should be strived for. The advantage of a marginal analysis based on the classification or the evaluation is that the number of variables $s$ of the result is reduced. In fact, by aggregating the different $\beta_{k}$ into a limited number of scores, the quest for options for improvement is further facilitated. An additional advantage is that the risk of roll off to other aspects is reduced. A 
practical problem, however, is that the construction of weighting factors for environmental problems is very awkward.

The method presented is not limited to the assessment of environmental aspects; the vector $b$ may contain any quantitative item that is relevant in product-related chain management, e.g., costs. A combined financial and environmental analysis is also possible.

The presentation of large amounts of data still remains one of the problems. A number of 100 processes (and thus 100 economic entities) and 50 environmental entities seems to be normal practice. The marginal analysis proposed above offers good opportunities to reduce the efforts of an ad hoc procedure and gives systematic results. Perhaps a hierarchy of elements of the marginal matrix in decreasing order, as in Table 2, could be useful. It clarifies in an instance the bunch of numbers underlying them. The potency of this method is clear; one obtains a presentation of the most important options for the improvement of the environmental performance of a product.

The usage of a reliability matrix should be supported in order to force LCA researchers to face the problem of the influence of unreliable data on the result. In some cases this may necessitate more carefully balanced claims on cleaner products. The marginal matrix may be used to identify the most critical data.

It is hoped that the development of LCA, in combination with a dynamic approach as with the marginal matrix, will lead to a continuous development of environmentally friendlier processes and products. Obviously, the broader context of sustainability should be kept in mind. This implies that the functional unit itself - that what is to be consumed - should be brought up for discussion, not in analysis, but in an assessment.

\section{Acknowledgement}

The author wishes to acknowledge Jeroen Guinée, Paul Mulder and Anneke Wegener Sleeswijk for criticizing parts of the manuscript. The referees made some valuable remarks con- cerning the connection between life cycle assessment and economic input-output analysis.

\section{Appendix}

The derivation of the marginal matrix requires some differential calculus. In this appendix, the expressions are elaborated. The problem is defined as finding $\Delta \beta_{k}\left(\Delta a_{j i}\right)$. Since $\beta_{k}$ depends on $a_{j i}$ via $p_{l}(l=1, \ldots, q)$, one has to first order

$\Delta \beta_{k}\left(\Delta a_{j i}\right)=\frac{\partial \beta_{k}}{\partial a_{j i}} \Delta a_{j i}=\sum_{l=1}^{q} \frac{\partial \beta_{k}}{\beta p_{l}} \frac{\partial p_{l}}{\partial a_{j i}} \Delta a_{j i}$.

From Eq. 3 for $\beta_{k}$ one finds

$\frac{\partial \beta_{k}}{\partial p_{l}}=b_{k} l$

and from Eq. 2 for $p_{i}$

$\frac{\partial p_{l}}{\partial a_{j i}}=\frac{-p_{l}}{\operatorname{det}(A)} \frac{\partial \operatorname{det}(A)}{\partial a_{j i}}+\frac{1}{\operatorname{det}(A)} \frac{\partial \operatorname{det}\left(A^{l}\right)}{\partial a_{j i}}$.

This formula contains the derivative of a determinant. A determinant can be expanded in terms of so-called minors $\boldsymbol{A}_{j i}$ defined by the matrix $A$ with the $j$ th row and the $i$ th column deleted, such that for any choice of $i$

$\operatorname{det}(A)=\sum_{j=1}^{r}(-1)^{j+i} a_{j i} \operatorname{det}\left(\boldsymbol{A}_{j i}\right)$.

Since $A_{m i}$ does not depend on $a_{j i}$, it follows that

$$
\begin{aligned}
\frac{\partial \operatorname{det}(A)}{\partial a_{j i}}= & \sum_{m=1}^{r}(-1)^{m+i} \frac{\partial a_{m i}}{\partial a_{j i}} \operatorname{det}\left(A_{m i}\right) \\
& +\sum_{m=1}^{r}(-1)^{m+i} a_{m i} \frac{\partial \operatorname{det}\left(A_{m i}\right)}{\partial a_{j i}} .
\end{aligned}
$$

Since

$\frac{\partial a_{m i}}{\partial a_{j i}}=\delta_{m j}$,

where $\delta_{m j}$ is the Kronecker-delta ( 1 when $m=j$; 0 otherwise), and

$$
\frac{\partial \operatorname{det}\left(A_{m i}\right)}{\partial a_{j i}}=0
$$


one derives that

$\frac{\partial \operatorname{det}(\boldsymbol{A})}{\partial a_{j i}}=(-1)^{j+i} \operatorname{det}\left(\boldsymbol{A}_{j i}\right)$.

More complicated is

$$
\begin{aligned}
\frac{\partial \operatorname{det}\left(A^{l}\right)}{\partial a_{j i}}= & \sum_{m=1}^{r}(-1)^{m+i} \frac{\partial a_{m i}^{l}}{\partial a_{j i}} \operatorname{det}\left(A_{m i}^{l}\right) \\
& +\sum_{m=1}^{r}(-1)^{m+i} a_{m i}^{l} \frac{\partial \operatorname{det}\left(A_{m i}^{l}\right)}{\partial a_{j i}}
\end{aligned}
$$

Since

$a_{m i}^{l}= \begin{cases}\alpha_{m} & (\text { if } i=l) \\ a_{m i} & \text { (otherwise) }\end{cases}$

one has

$$
\frac{\partial a_{m i}^{l}}{\partial a_{j i}}= \begin{cases}0 & \text { (if } i=l) \\ \delta_{j m} & \text { (otherwise) }\end{cases}
$$

\section{Furthermore}

$$
\frac{\partial \operatorname{det}\left(A_{m i}^{l}\right)}{\partial a_{j i}}=0 \text {. }
$$

The expression is now simplified as

$$
\frac{\partial \operatorname{det}\left(A^{l}\right)}{\partial a_{j i}}= \begin{cases}0 & \text { (if } i=l) \\ (-1)^{j+i} \operatorname{det}\left(A_{j i}^{l}\right) & \text { (otherwise) }\end{cases}
$$

The equation gives, when substituted in Eq. 13:

$$
\begin{aligned}
\frac{\partial p_{l}}{\partial a_{j i}}= & -\frac{p_{l}}{\operatorname{det}(A)}(-1)^{j+i} \operatorname{det}\left(A_{j i}\right) \\
& + \begin{cases}0 & (\text { if } i=l) \\
\frac{(-1)^{j+i} \operatorname{det}\left(A_{j i}^{l}\right)}{\operatorname{det}(A)} & \text { (otherwise) }\end{cases}
\end{aligned}
$$

This gives for $\Delta \beta_{k}$

$$
\begin{aligned}
\frac{\partial \beta_{k}}{\partial a_{j i}}= & -\frac{(-1)^{j+i} \operatorname{det}\left(A_{j i}\right)}{\operatorname{det}(A)} \sum_{l=1}^{q}\left(b_{k l} p_{l}\right) \\
& +\frac{(-1)^{j+i}}{\operatorname{det}(A)} \sum_{\substack{l=1 \\
l \neq i}}^{q}\left(b_{k l} \operatorname{det}\left(A_{j i}^{l}\right)\right)
\end{aligned}
$$

which is by definition (see Eq. 3) equivalent to

$$
\begin{aligned}
\frac{\partial \beta_{k}}{\partial a_{j i}}= & -\frac{(-1)^{j+i} \operatorname{det}\left(A_{j i}\right)}{\operatorname{det}(A)} \beta_{k} \\
& +\frac{(-1)^{j+i}}{\operatorname{det}(A)} \sum_{\substack{l=1 \\
l \neq i}}^{q}\left(b_{k l} \operatorname{det}\left(A_{j i}^{l}\right)\right)
\end{aligned}
$$

which proves Eq. 5 .

For a change in $\alpha_{j}$ a similar approach can be followed:

$\Delta \beta_{k}\left(\Delta \alpha_{j}\right)=\frac{\partial \beta_{k}}{\partial \alpha_{j}} \Delta \alpha_{j}=\sum_{i=1}^{q} \frac{\partial \beta_{k}}{\partial p_{i}} \frac{\partial p_{i}}{\partial \alpha_{j}} \Delta \alpha_{j}$,

in which

$\frac{\partial p_{i}}{\partial \alpha_{j}}=\frac{-p_{i}}{\operatorname{det}(\mathbf{A})} \frac{\partial \operatorname{det}(\mathbf{A})}{\partial \alpha_{j}}+\frac{1}{\operatorname{det}(\mathbf{A})} \frac{\partial \operatorname{det}\left(\mathbf{A}^{i}\right)}{\partial \alpha_{j}}$.

The partial derivative of $p_{i}$ with respect to $\alpha_{j}$ is easier than the one with respect to $a_{j i}$ :

$\frac{\partial \operatorname{det}\left(A^{i}\right)}{\partial \alpha_{j}}=(-1)^{j+i} \operatorname{det}\left(A_{j i}\right)$

and

$$
\frac{\partial \operatorname{det}(\boldsymbol{A})}{\partial \alpha_{j}}=0
$$

so that substitution yields

$$
\frac{\partial p_{i}}{\partial \alpha_{j}}=\frac{1}{\operatorname{det}(A)}(-1)^{j+i} \operatorname{det}\left(A_{j i}\right) \text {. }
$$

This yields for $\partial \beta_{k} / \partial \alpha_{j}$ :

$$
\frac{\partial \beta_{k}}{\partial \alpha_{j}}=\sum_{i=1}^{q} \frac{(-1)^{j+i} \operatorname{det}\left(A_{j i}\right)}{\operatorname{det}(A)} b_{k i}
$$

so that Eq. 6 is derived.

Much more trivial are changes in $b_{n i}$

$$
\frac{\partial \beta_{k}}{\partial b_{n i}}=p_{i} \delta_{i n}
$$

leading to Eq. 7, and in $\beta_{n}$

$$
\frac{\partial \beta_{k}}{\partial \beta_{n}}=\delta_{n k}
$$

leading to Eq. 8. 


\section{References}

Anonymous, 1992. Product Life Cycle Assessment - Principles and Methodology. Nord, Copenhagen, $288 \mathrm{pp.}$

Ayres, R.U. and Rod, S.R., 1986. Patterns and pollution in the Hudson-Raritan basin. Environment, 28: 14-20 and 39-43.

Commons, M. and Perring, C., 1992. Towards an ecological economics of sustainability. Ecol. Econ., 6: 7-34.

Duchin, F., 1992. Industrial input-output analysis: implications for industrial ecology. Proc. Natl. Acad. Sci. USA, 89: 851-855.

Fava, J.A., Denison, R., Jones, B., Curran, M.A., Vigon, B., Selke, S. and Barnum, J. (Editors), 1991. A Technical Framework for Life-cycle Assessment. SETAC, Washington, $134 \mathrm{pp}$.

Fraunhofer-Institut für Lebensmitteltechnologie und Verpackung, Gesellschaft für Verpackungsmarktforschung, Institut für Energie- und Umweltforschung, 1991. Umweltprofile von Packstoffen und Packmitteln, Methode (Entwurf). ILV, München, 99 pp. (in German).

Goedkoop, M.J., 1991. SIMAPRO manual. Computer program for the assessment of the environmental impacts of products. Pré Consultants, Amersfoort, $25 \mathrm{pp}$.

Guinée, J.B., Udo de Haes, H.A. and Huppes, G., 1993. Quantitative life cycle assessment of products: goal definition and inventory. J. Cleaner Prod., 1: 3-13.
Heijungs, R., Guinée, J.B., Huppes, G., Lankreijer, R.M., Udo de Haes, H.A. Wegener Sleeswijk, A., Ansems, A.M.M., Eggels, P.G., van Duin, R. and de Goede. II.P., 1992. Environmental life cycle assessment of products. I. Guide, October 1992, II. Backgrounds, October 1992. CML, Leiden, $226 \mathrm{pp}$.

Heijungs, R., Mulder P.A.A. and Guinée J.B., in preparation.

Hocking, M.B., 1991. Paper versus polystrene: a complex choice. Science, 251: 504-505.

Huppes, G., 1991. Allocating impacts of multiple economic processes in LCA. In: SETAC-Europe workshop. Lifecycle Assessment. SETAC, Brussels.

Leontief, W., 1986. Input-output analysis. In: M.B. Bever (Editor), Encyclopedia of Materials, Science and Engineering. Pergamon, Oxford, pp. 2339-2349.

Lübkert, B., Virtanen, Y., Mühlberger, M., Ingman, J., Vallance, B. and Alber, S., 1991. Life-cycle analysis. IDEA, an international database for ecoprofile analysis. A tool for decision makers. Working paper, September 1991. IIASA, Laxenburg, $174 \mathrm{pp}$.

Patel, C.K.N., 1992. Industrial ecology. Proc. Natl. Acad. Sci. USA, 89: 798-799.

SETAC-Europe workshop, 1992. Life-cycle Assessment. SETAC, Brussels, $146 \mathrm{pp}$

Vigon, B.W., Tolle, D.A., Cornaby, W., Latham, H.C., Harrison, C.L., Boguski, T.L., Hunt, R.G. and Sellers, J.D., 1992. Product Life-cycle Assessment: Inventory Guidelines and Principles. EPA, Cincinatti, $108 \mathrm{pp}$. 